\title{
Guillain-Barré syndrome in Taiwan: a clinical study of 167 patients
}

\author{
Rong-Kuo Lyu, Lok-Ming Tang, Shaw-Yi Cheng, Wen-Chuin Hsu, Sien-Tsong Chen
}

\begin{abstract}
Objective-To identify clinical characteristics of various forms of Guillain-Barré syndrome in Taiwan.

Methods-The clinical and electrophysiological data of 167 consecutive patients with Guillain-Barré syndrome admitted to Chang Gung Memorial Hospital, a general paediatric and adult hospital in Taiwan, were reviewed.

Results-Analysis of age distribution disclosed a high incidence (21\%) among patients under the age of 10 years. Seasonal preponderance in Spring (March to May) was found. Utilising clinical and electrophysiological data, these $167 \mathrm{pa}$ tients with Guillain-Barré syndrome were subclassified; 82 (49\%) had acute inflammatory demyelinating polyradiculoneuropathy (AIDP), 32 (19\%) had Fisher syndrome (FS), and six (4\%) had axonal forms of Guillain-Barré syndrome. The remaining $47(28 \%)$ patients were unclassified. Patients with AIDP and FS had many common clinical features, including seasonal distribution, history of preceding illness, sensory abnormalities, cranial nerve involvement except for extraocular motor nerves, and albuminocytological dissociation on examination of CSF. Follow up study on 145 patients disclosed that $127(87 \%)$ recovered satisfactorily, 14 $(10 \%)$ were persistently disabled, and four (3\%) died during admission to hospital. Clinical features associated with poor outcome (persistent disability or death) were requirement for mechanical ventilation, a low mean compound muscle action potential amplitude ( $\leqslant 10 \%$ of the lower limit of normal), and age greater than 40 years.

Conclusion-Guillain-Barré syndrome in Taiwan showed a peculiar age and seasonal distribution and a high frequency of FS not seen in other series. Given that patients with AIDP and FS had many common clinical features, AIDP and FS may have similar underlying pathological mechanisms.
\end{abstract}

(F Neurol Neurosurg Psychiatry 1997;63:494-500)

Keywords: Guillain-Barré syndrome; clinical features; Taiwan

Based on pathological ${ }^{1}$ and electrophysiological studies, ${ }^{23}$ Guillain-Barré syndrome has been considered to be primarily a demyelinating disease which is synonymous with acute inflammatory demyelinating polyradiculoneuropathy (AIDP). After Fisher's classic description, ${ }^{4}$ Fisher syndrome (FS), which is characterised by the triad of ataxia, areflexia, and ophthalmoplegia, has been recognised as a clinical variant of Guillain-Barré syndrome. The concept of axonal Guillain-Barré syndrome was first raised by Feasby et $a l,{ }^{5}$ who found early axonal degeneration of motor and sensory fibres in five patients with clinically defined Guillain-Barré syndrome. This pattern of Guillain-Barré syndrome, termed acute motor-sensory axonal neuropathy (AMSAN), is usually associated with a worse prognosis than demyelinating Guillain-Barré syndrome. Studies in northern China have identified another form of axonal Guillain-Barré syndrome, termed acute motor axonal neuropathy (AMAN). ${ }^{67}$ Thus information learned from recent studies of Guillain-Barré syndrome suggests that it may be subclassified into several forms, and proposed relations among these forms of Guillain-Barré syndrome have been suggested. ${ }^{8}$

Guillain-Barré syndrome is a worldwide disease. ${ }^{9}$ There has been no systematic study of the syndrome in Taiwan, although multiple reports exist from mainland China showing a characteristic pattern suggesting predominantly motor axonal involvement. ${ }^{67}$ The purpose of this study was to review cases of Guillain-Barré syndrome admitted to Chang Gung Memorial Hospital during a period of 16 years to ascertain whether a distinct pattern of the syndrome exists in Taiwan. In addition, we paid special attention to the secular trend of the disease, and the clinical and laboratory differences among subgroups of patients.

\section{Patients and methods}

We reviewed the medical records of all patients discharged from Chang Gung Memorial Hospital with a diagnosis of Guillain-Barré syndrome between January 1980 and December 1995. All patients were treated at Chang Gung Memorial Hospital, a 3500 bed teaching hospital located in the northern part of Taiwan and also a tertiary referring medical centre for the whole country. The population in this island is estimated to be 21 million. We recorded data on age, sex, preceding events, date of onset of disease, clinical manifestations including initial symptoms and neurological findings during the course, results of CSF study, and specific treatments including steroids, plasmapheresis, and intravenous immunoglobulin (IVIg). Only data of the first CSF study were used for analysis. We also registered 
Table 1 Demographic features and preceding events in patients with various forms of Guillain-Barré syndrome

\begin{tabular}{lllll}
\hline & AIDP $(n=82)$ & $F S(n=32)$ & Axonal forms $(n=6)$ & Unclassified $(n=47)$ \\
\hline Mean age (y) & $34.6^{\star}$ & 45.0 & 44.3 & 28.7 \\
Sex: (male/female) & $66 / 34$ & $56 / 44$ & $100 / 0$ & $66 / 34$ \\
Preceding events & 72 & 56 & 50 & 68 \\
$\quad$ Upper respiratory tract infection & 55 & 56 & 17 & 57 \\
$\quad$ Fever and chill & 2 & 0 & 0 & 2 \\
Acute gastroenteritis & 2 & 0 & 0 & 0 \\
Acute conjunctivitis & 0 & 0 & 17 & 2 \\
Identifiable viral infections & 7 & 0 & 0 & 0 \\
$\quad$ Bacterial infections & 4 & 0 & 17 & 0 \\
Traffic accident & 1 & 0 & 0 & 6 \\
\hline
\end{tabular}

$\star \mathrm{P}<0.05 v \mathrm{FS}$ subgroup.

AIDP = Acute inflammatory demyelinating polyradiculoneuropathy; FS = Fisher syndrome. All the values (except those for mean age) are given as a percentage.

the findings of electrophysiological studies, including distal motor, sensory, and $\mathrm{F}$ wave latencies, amplitudes of evoked compound muscle action potentials (CMAPs) and sensory nerve action potentials (SNAPs), and motor and sensory conduction velocities. The median interval between onset of neuropathy and performance of the first electrophysiological study was 12.5 days.

At the time of their maximal deficits during admission to hospital, patients were graded using a disability scale modified from Hughes et al. ${ }^{10}$ Grade 0 is defined by normal functional state without neurological deficits; grade 1, minor symptoms or signs but being able to do manual work; grades 2 and 3, ambulation without or with assistance respectively; grade 4, chair or bed bound; grade 5, requiring mechanical ventilator; and grade 6 , death.

Based on initial electrophysiological findings, patients were classified as having AIDP when there was evidence of demyelination in at least two tested nerves. ${ }^{7}$ Abnormal values suggestive of demyelination in nerve conduction studies were defined as follows: ${ }^{3}>120 \%$ of upper limit of normal in distal latency, $<60 \%$ in proximal/distal CMAP amplitude ratio, $<85 \%$ of lower limit of normal in conduction velocity, and $>120 \%$ of upper limit of normal in $F$ wave latency. Patients were classified as having axonal forms of Guillain-Barré syndrome (AMSAN or AMAN) if there was no electrophysiological evidence of demyelination as defined above, together with a decrease of CMAP or SNAP amplitudes to less than $80 \%$ of lower limit of normal in at least two tested nerves. ${ }^{7}$ Patients who were not studied electrophysiologically or whose data did not conform to either category were considered unclassifiable. A diagnosis of FS was made in patients who presented with the triad of ataxia, areflexia, and ophthalmoplegia. ${ }^{4}$ Coincidence of minimal limb weakness did not exclude the diagnosis of FS. ${ }^{11}$ In addition, mean CMAP amplitude from distal stimulation was calculated. ${ }^{12}$

Outcome was determined according to the functional recovery of the patients. A good outcome was defined by a satisfactory recovery and resumption of a normal functional life (disability grade of 0 or 1 ). Poor outcome was defined by persistent disability or death during admission to hospital (disability grade of 2 or greater).
Student's $t$ test and two sided Fisher's exact test were used for comparative analysis. Seasonal preponderance was tested by goodness of fit $\chi^{2}$ test, assuming a null hypothesis of no seasonal variation. Multivariable logistic regression was used to analyse the effect of plasmapheresis or IVIg on long term outcome, adjusted for age and the maximal deficits during admission to hospital.

\section{Results}

During the study period, 167 patients who fulfilled the diagnostic criteria for Guillain-Barré syndrome ${ }^{13}$ were identified. According to the clinical and electrophysiological findings, 82 patients (49\%) had AIDP (hereafter AIDP subgroup), 32 (19\%) had FS (FS subgroup), and six (4\%) had axonal forms of GuillainBarré syndrome (axonal subgroup). In this study, $47(28 \%)$ patients were unclassified, of whom 24 had no electrophysiological studies and two had absent responses in all tested nerves; the remaining 21 patients had mild electrophysiological abnormalities and hence they could not be subgrouped into either AIDP or axonal forms.

AGE, SEX, AND SEASONAL DISTRIBUTIONS

The mean (SD) age of all 167 patients was 35.3 (23.7) years (range 1.4 to 77 years) with the highest frequency ( $21 \%$ of all patients) occurring in children under the age of 10 years (fig 1). This high rate in children under age 10 occurred in the AIDP subgroup but not in the FS or axonal subgroups. A total of $109(65 \%)$ patients were male ( $95 \%$ confidence interval $57 \%-72 \%$, binomial exact method), which significantly outnumbered females. The male predominance occurred only in the AIDP and axonal subgroups (table 1). Seasonal preponderance was found in both the AIDP and FS subgroups: $44 \%$ of patients with AIDP $(\mathrm{P}=0.0012)$ and $47 \%$ of patients with $\mathrm{FS}$ $(\mathrm{P}=0.023)$ developed the disease in Spring (March to May, fig 2).

PRECEDING EVENTS

Various preceding events within one month before onset of the illness were noted in 112 (67\%) patients. All but one event were infectious diseases (table 1), mostly nonspecific upper respiratory tract infection, which occurred in 91 (54\%) patients. Identifiable viral infections included herpes zoster, Rubella, 


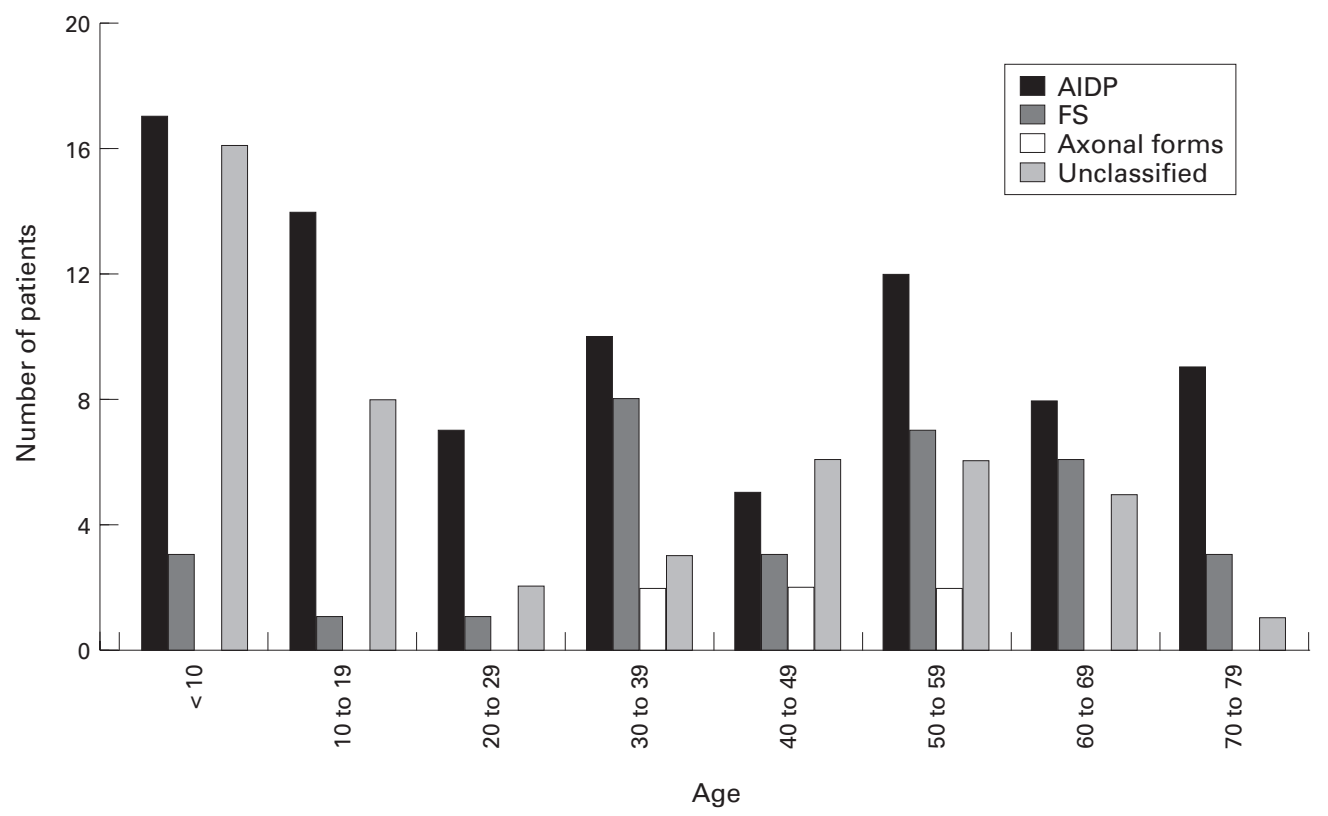

Figure 1 Age distribution of Guillain-Barré syndrome. AIDP=acute inflammatory demyelinating polyradiculoneuropathy; FS=Fisher syndrome.

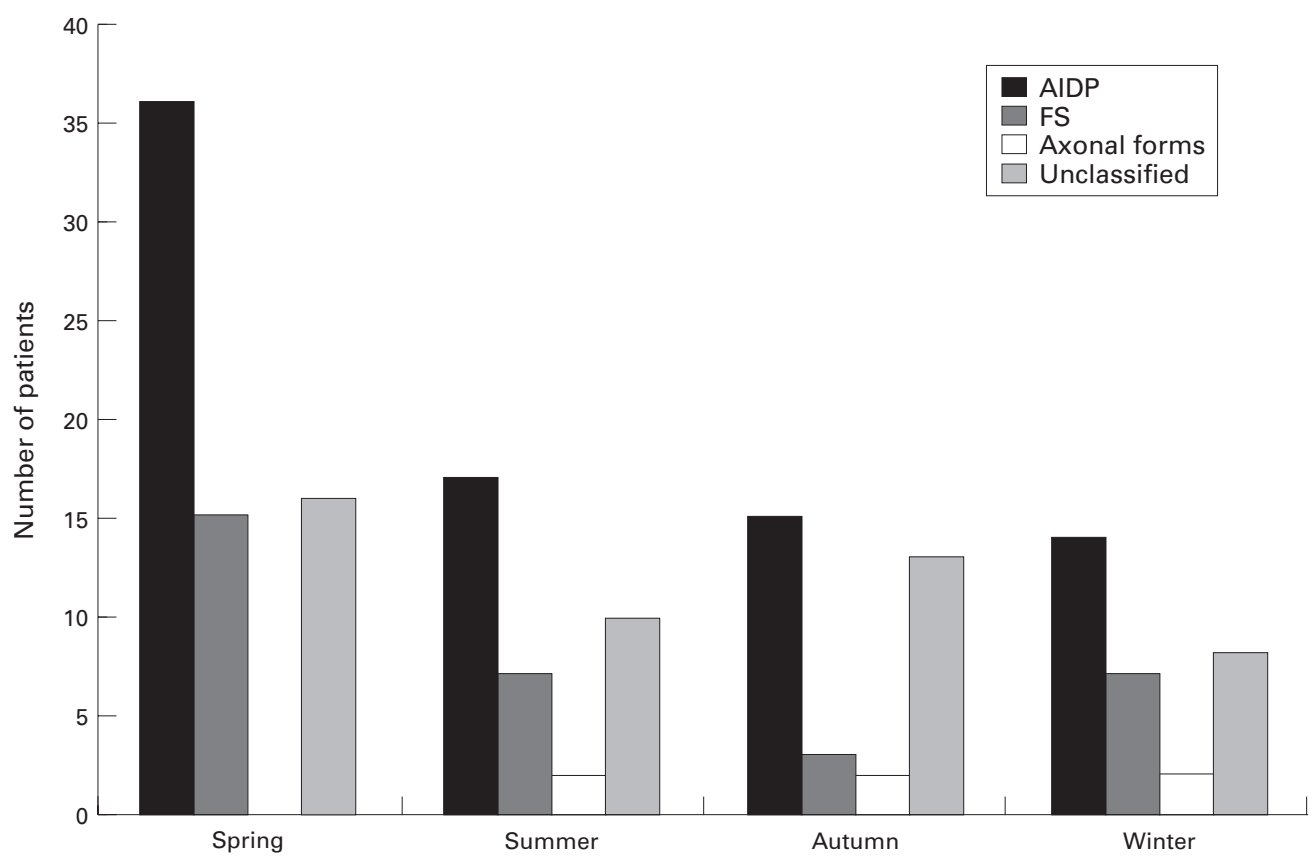

Figure 2 Seasonal distribution of Guillain-Barré syndrome. AIDP=acute inflammatory demyelinating polyradiculoneuropathy; $F S=$ Fisher syndrome

and chicken pox each in two patients and acute viral hepatitis in one. Bacterial infections included gonorrhoea, mycoplasma pneumonia, acute tonsillitis with Acinectobacter, and urinary tract infection with Escherichia coli each in one patient. Vaccination was not an antecedent event in our patients. The median interval between the onset of preceding events and the onset of symptoms was eight days (range 1 to 30 days).

CLINICAL FEATURES

The most frequent initial symptoms in the AIDP and axonal subgroups were numbness or paresthaesia and limb weakness whereas in the
FS subgroup, these were blurred vision, dizziness, and ataxia (table 2). Ptosis and facial weakness as initial symptoms occurred only in patients with FS. The median evolving time from the onset of illness to the nadir in all patients was seven days (range 1 to 42 days). Only one patient had a clinical course evolving more than four weeks. The progressive phase in the patient was 42 days; he was still considered to be a case of Guillain-Barré syndrome because of a typical clinical feature with monophasic course, nearly complete recovery, and no recurrence after a seven year follow up.

Table 3 summarises the clinical features during the course of the disease. Reduced or 
Table 2 Initial symptoms and evolving times in patients with various forms of Guillain-Barré syndrome

\begin{tabular}{lllll}
\hline Initial symptoms & $\begin{array}{l}\text { AIDP } \\
(n=82)\end{array}$ & $\begin{array}{l}F S \\
(n=32)\end{array}$ & $\begin{array}{l}\text { Axonal forms } \\
(n=6)\end{array}$ & $\begin{array}{l}\text { Unclassified } \\
(n=47)\end{array}$ \\
\hline Numbness or paraesthesia & $50^{\star \star \star}$ & 16 & 67 & 30 \\
Limb weakness & $44^{\star \star \star}$ & 6 & 33 & 62 \\
Muscle pain or soreness & 10 & 0 & 0 & 6 \\
Bulbar dysfunction & 12 & 19 & 0 & 2 \\
Ataxia & $2^{\star \star \star}$ & 28 & 0 & 6 \\
Dizziness & $2^{\star \star \star}$ & 28 & 0 & 2 \\
Blurred vision & $1^{\star \star \star}$ & 34 & 0 & 2 \\
Headache & $1^{\star}$ & 13 & 0 & 4 \\
Back pain & 1 & 3 & 17 & 2 \\
Ptosis & $0^{\star}$ & 9 & 0 & 0 \\
Facial weakness & 0 & 3 & 0 & 0 \\
Median evolving duration & & & & 6 \\
$\quad$ (days) & 8 & 5.5 & 3 & \\
\hline
\end{tabular}

${ }^{\star} \mathrm{P}<0.05 ; \star \star \star \mathrm{P}<0.001 v$ FS subgroup.

AIDP $=$ Acute inflammatory demyelinating polyradiculoneuropathy; FS = Fisher syndrome. All the values (except those for median evolving duration) are given as a percentage.

Table 3 Clinical features in patients with various forms of Guillain-Barré syndrome

\begin{tabular}{lcccc}
\hline Clinical features & $\begin{array}{c}\text { AIDP } \\
(n=82)\end{array}$ & $\begin{array}{c}F S \\
(n=32)\end{array}$ & $\begin{array}{l}\text { Axonal forms } \\
(n=6)\end{array}$ & $\begin{array}{l}\text { Unclassified } \\
(n=47)\end{array}$ \\
\hline Reduced or absent tendon & & & & \\
$\quad$ reflexes & 100 & 100 & 100 & 100 \\
Limb weakness & $99^{\star \star \star}$ & 25 & 100 & 100 \\
Sensory disturbance & 65 & 59 & 67 & 60 \\
$\quad$ Sensory symptoms & 54 & 34 & 67 & 49 \\
Sensory signs & 52 & 50 & 17 & 36 \\
External ophthalmoplegia & $17^{\star \star \star}$ & 100 & 17 & 21 \\
Trigeminal dysfunction & 15 & 16 & 0 & 11 \\
Facial weakness & 49 & 50 & 17 & 34 \\
Bulbar weakness & 52 & 59 & 17 & 47 \\
Respiratory failure & $32^{\star \star \star}$ & 0 & 17 & 23 \\
Autonomic dysfunction & $27^{\star \star}$ & 3 & 0 & 34 \\
Sinus tachycardia & 20 & 3 & 0 & 23 \\
Bladder dysfunction & 11 & 3 & 0 & 11 \\
Sinus bradycardia & 5 & 0 & 0 & 2 \\
Sweating disturbance & 4 & 0 & 0 & 2 \\
Hypertension & 5 & 0 & 0 & 0 \\
Hypotension & 1 & 0 & 0 & 0 \\
Ataxia & $13^{\star \star \star}$ & 100 & 33 & 26 \\
Ptosis & $10^{\star \star \star}$ & 59 & 17 & 11 \\
Limb dysmetria & $4^{\star \star \star}$ & 44 & 17 & 6
\end{tabular}

$\star \star \mathrm{P}<0.01 ;{ }^{\star \star \star} \mathrm{P}<0.001 v$ FS subgroup.

AIDP = Acute inflammatory demyelinating polyradiculoneuropathy; FS = Fisher syndrome. All the values are given as a percentage.

Table 4 Factors associated with outcome in patients with Guillain-Barré syndrome

\begin{tabular}{lccl}
\hline Outcome & Good & Poor & P value \\
\hline Requirement for mechanical ventilation & $25 / 127(20 \%)$ & $10 / 18(56 \%)$ & $<0.01$ \\
Mean CMAP amplitude $\leqslant 10 \%$ of LLN & $6 / 107(6 \%)$ & $5 / 16(31 \%)$ & $<0.01$ \\
Age $>40$ y & $52 / 127(41 \%)$ & $12 / 18(67 \%)$ & $<0.05$ \\
\hline
\end{tabular}

$\mathrm{CMAP}=$ Compound muscle action potentials; $\mathrm{LLN}=$ lower limit of normal.

absent reflexes was a universal finding. All but one patient in the AIDP subgroup and all patients in the axonal subgroup had limb weakness. In the FS subgroup, eight (25\%) patients had minimal limb weakness. Truncal ataxia rather than limb weakness was the major cause of gait disturbance in these eight patients. Sensory disturbance occurred in 59 to $67 \%$ of patients in all three subgroups. Cranial nerve involvement except for ocular motor nerves occurred with similar frequency in both AIDP and FS subgroups. Respiratory failure and autonomic dysfunction occurred predominantly in AIDP, whereas ataxia, ptosis, and limb dysmetria occurred predominantly in FS (table 3). At the time of their maximal deficits, $8(10 \%)$ patients in the AIDP subgroup reached grade $2,15(18 \%)$ grade $3,33(40 \%)$ grade 4 , and $26(32 \%)$ grade 5 . By contrast, patients with FS generally reached a less severe disability at their nadir: six $(19 \%)$ in grade 2 ,
$21(66 \%)$ grade 3 , five (16\%) grade 4 , and none in grade 5 . In the axonal subgroup, three $(50 \%)$ patients were in grade 3, two $(33 \%)$ grade 4 , and one $(17 \%)$ grade 5 . Respiratory failure was found in $26(32 \%)$ patients in the AIDP subgroup, one $(17 \%)$ in the axonal subgroup, and $11(23 \%)$ in the unclassified subgroup (table 3 ). The median duration of mechanical ventilation of the 36 out of 38 patients (data being missed in two patients) was 10 days (range 2 to 70 days, fig 3 ).

\section{CEREBROSPINAL FLUID STUDIES}

A lumbar puncture was performed on 135 patients within two months of the onset of illness, with a median of nine days (range 1 to 60 days). The CSF protein concentration (mean 95.2 , range $6-580 \mathrm{mg} / \mathrm{dl}$ ) was raised ( $\geqslant$ $45 \mathrm{mg} / \mathrm{dl}$ ) in 96 patients (71\%). The frequency of raised CSF protein concentration was $52 \%$ when the CSF study was done in the first week of illness, $78 \%$ in the second week, and 93\% beyond the second week. The CSF cell count (mean 3.0 cells $/ \mathrm{mm}^{3}$ ) was normal ( $<10$ cells/ $\mathrm{mm}^{3}$ ) in 123 patients $(91 \%)$ and was slightly raised in $12\left(9 \%\right.$, range $12-27$ cells $\left./ \mathrm{mm}^{3}\right)$. None of the patients with raised cell counts had human immunodeficiency virus infection. Two patients in the first week of illness and five each in the second week and beyond the second week had CSF pleocytosis. In total, 84 patients $(62 \%)$ showed albuminocytological dissociation (raised protein concentration without pleocytosis) on CSF examination.

Data for CSF were available in 67 patients with AIDP, 26 with FS, and five with the axonal form of Guillain-Barré syndrome. In these three subgroups, raised protein concentration was found in $54(81 \%), 15(58 \%)$, and five $(100 \%)$ patients; abnormal cell count in five $(7 \%)$, two $(8 \%)$, and two $(40 \%)$ patients; and albuminocytological dissociation in 49 $(73 \%), 13(50 \%)$, and three $(60 \%)$ patients respectively.

SPECIFIC TREATMENTS, OUTCOMES, AND PROGNOSTIC FACTORS

The average duration of admission to hospital for all patients was 22.8 days (range 2 to 147 days). In addition to general medical management, $93(55 \%)$ patients received specific treatments: 55 (59\%) steroids alone, 27 (29\%) plasmapheresis alone, one (1\%) IVIg alone, nine $(10 \%)$ steroids together with plasmapheresis or IVIg, and one (1\%) plasmapheresis followed by IVIg. Before 1989, steroids were the main specific treatment and were used in 46 (96\%) of the 48 patients who received specific treatment. Since then, plasmapheresis and IVIg were often employed and $33(73 \%)$ of the 45 patients had either modality as their specific treatment.

Twenty five $(78 \%)$ of the 32 patients with plasmapheresis and six (86\%) of the seven patients with IVIg including the one whose condition remained unchanged for two weeks after prior plasmapheresis improved at least one disability grade within 14 days after the 
treatment was given. The median duration from the time of treatment to the time of improvement was five and 6.5 days for those who received plasmapheresis and IVIg respectively. Meanwhile, only half of the 64 patients who were treated with steroids improved within two weeks after treatment.

Follow up study was done on 145 patients. The median follow up period was 12.8 months (range 3 months to 12 years). A good outcome with normal functional life was noted in 127 $(87 \%)$ patients. A poor outcome was found in $18(13 \%)$ patients, including nine $(12 \%)$ with AIDP, two $(7 \%)$ with FS, one $(17 \%)$ with the axonal form of Guillain-Barré syndrome, and six $(17 \%)$ unclassified. Persistent ataxia was the cause of functional disability in the two patients with FS who recovered unsatisfactorily. Four patients $(3 \%)$ died during admission to hospital: three with AIDP and one unclassified. The causes of death included sudden cardiac arrest, acute pulmonary embolism, pneumonia, and ruptured tension pneumothorax. The factors associated with a poor outcome included requirement of mechanical ventilation, a low mean CMAP amplitude (10\% or less of lower limit of normal) and age greater than 40 years (table 4). Sex, preceding infectious illness, time to reach nadir, bulbar paralysis, evolving pattern of weakness, and CSF protein concentration were not prognostic factors. Neither plasmapheresis nor IVIg treatment would reduce the duration of ventilator dependence time, time to onset of motor recovery, or admission to hospital time in our patients. In addition, long term outcome was not affected by these two treatment modalities, independent of the maximal deficits during admission to hospital.
COMPARATIVE ANALYSIS BETWEEN DIFFERENT GUILLAIN-BARRÉ SYNDROME SUBGROUPS Compared with the AIDP subgroup, the FS subgroup was older (table 1), and had a shorter duration of stay in hospital (mean, $15.6 v 25.5$ days, $\mathrm{P}=0.001$ ) and a lower $\mathrm{CSF}$ protein concentration (mean, $61.3 \vee 116.0 \mathrm{mg} / \mathrm{dl}$, $\mathrm{P}<0.001)$. The FS subgroup was often associated initially with ataxia, dizziness, blurred vision, headache, and ptosis, and lately with external ophthalmoplegia, ataxia, ptosis, and limb dysmetria. Besides, limb weakness and autonomic dysfunction occurred less often in patients with FS (table 3). Furthermore, patients with FS had a less severe disabling state at their maximal deficits (percentage of patients progressing to grade 4 or worse, $16 \% v$ $72 \%, \mathrm{P}<0.001$ ), and had no respiratory failure. The patients with axonal forms showed no distinguishing features from those with AIDP.

\section{Discussion}

Of the 167 patients with clinically defined Guillain-Barré syndrome, $32(19 \%)$ had FS. This high frequency of FS, a variant form of the syndrome, contrasts with a low frequency of $2 \%$ to $7 \%$ in series from the western world. ${ }^{11} 1415$ Axonal forms of Guillain-Barré syndrome, including AMAN and AMSAN, occurred rarely in our patients (4\%). This differs from the series from northern China, in which AMAN is often encountered. ${ }^{6}$

Over the past 40 years, most Guillain-Barré syndrome series from Europe and North America showed a higher incidence among patients over 40 years of age, with peaks of incidence variously in the fifth decade or later. ${ }^{11}{ }^{14} 16-20$ Although a higher incidence in young children was suggested occasionally, the

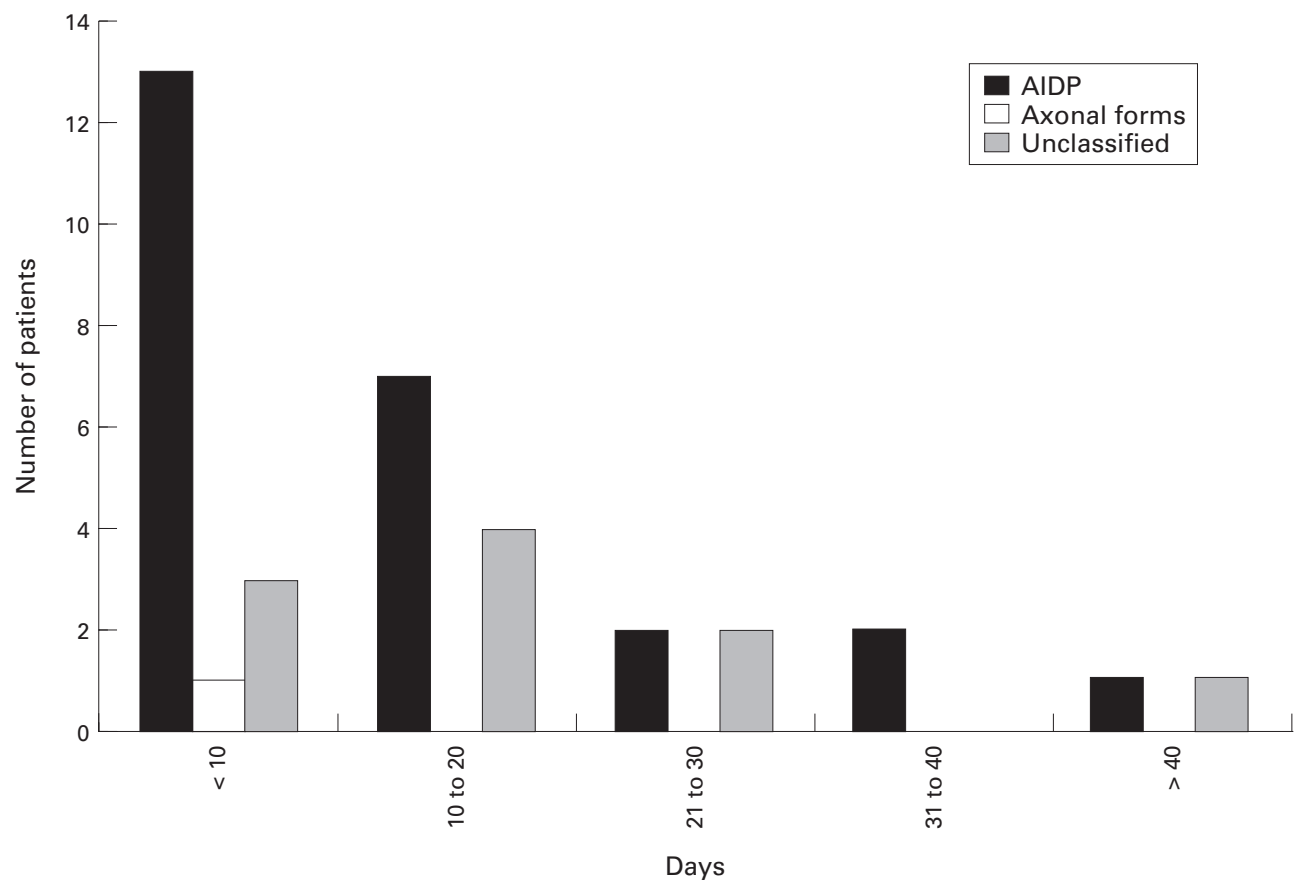

Figure 3 Duration of mechanical ventilation in the 36 patients with respiratory failure. AIDP=acute inflammatory demyelinating polyradiculoneuropathy. 
major peak incidence has always been in adults. ${ }^{21-23}$ Guillain-Barré syndrome occurring predominantly in children was reported recently from northern China, with $33 \%$ of the patients being under 7 years old. ${ }^{6}$ Our series showed a pattern of age distribution resembling that of northern China, with the highest frequency $(21 \%)$ occurring in children under the age of 10 years. However, this high frequency in children was found only in the AIDP subgroup. In both the FS and axonal subgroups, a higher frequency was noted in patients over the age of 30 .

Whereas seasonal trends did not occur in most series, ${ }^{11} 14162122$ a mild seasonal clustering was noted in the autumn and winter months, ${ }^{17} 1920$ and occasionally in the spring. ${ }^{23}{ }^{24}$ In the studies from northern China and Paraguay, a striking seasonal preponderance was found in the summer months. ${ }^{6}{ }^{25} \mathrm{In}$ our study, there was a minor seasonal clustering in the spring in both the AIDP and FS subgroups but not in the axonal subgroup. In the northern China study, Campylobacter jejuni infection was a risk factor. ${ }^{6}$ However, we could not correlate the seasonal predominance with preceding infectious diseases in our patients.

Preceding events were detectable in $67 \%$ of our patients, a figure close to other series of $40 \%$ to $82 \% .^{1114161819}$ In most series, nonspecific upper repiratory tract infection was usually the most common preceding event, followed by acute gastrointestinal illness, which occurred in $8 \%$ to $17 \%$ of patients. ${ }^{11} 141819$ Recently $C$ jejuni infection with or without preceding diarrhoeal illness has been recognised as an important event related to Guillain-Barré syndrome, especially in the axonal forms. ${ }^{715}$ In our series, upper repiratory tract infection was the most often encountered antecedent illness in both the AIDP and FS groups. Acute gastrointestinal illness occurred only in three patients $(2 \%)$ and none of them had an axonal form of Guillain-Barré syndrome.

Although FS has been recognised as a clinical variant of Guillain-Barré syndrome, ${ }^{411} \mathrm{sev}$ eral clinical case reports suggested that the cause of FS was a brainstem abnormality alone or in combination with peripheral nerve pathology. ${ }^{26-28}$ Evidence of CNS involvement in FS included drowsiness during the early stage of illness, ${ }^{26}$ ophthalmological signs of central origin such as mild ptosis in the presence of severe ophthalmoplegia, preserved Bell's phenomenon despite paralysis of upward gaze and horizontal dissociated nystagmus, ${ }^{27}{ }^{29}$ and abnormal imaging study in the brainstem. ${ }^{28}$ However, recent reviews on FS suggested that patients with various eye movement disorders could be explained by cranial nerve dysfunction alone, ${ }^{11}$ and those patients who were drowsy initially might represent another disorder rather than FS. ${ }^{30}$ In addition, serial neurophysiological studies have shown unequivocal evidence of peripheral nerve dysfunction in patients with FS. ${ }^{31} 32$

In the present study, none of our patients with FS presented with drowsiness or ophthalmological signs suggestive of central origin except one, who had transient horizontal dissociated nystagmus. Furthermore, many clinical features presented in patients with FS were similar to those in patients with AIDP. They were seasonal distribution, history of previous illness, sensory abnormalities including sensory loss on clinical examination, cranial nerve involvement except for extraocular motor nerves, and cell count and albuminocytological dissociation on CSF examination. In addition, two patients who developed typical AIDP later in their clinical course presented initially with asymmetric, partial ophthalmoplegia, bulbar weakness, and ataxia. Both patients were diagnosed as having FS on admission; complete tetraplegia and respiratory failure developed five and nine days later respectively. These clinical findings suggest that peripheral nerve dysfunction may be the main underlying mechanism in FS as that in AIDP.

Follow up studies showed that most of our patients recovered without appreciable neurological sequelae and resumed a normal life. The mortality in our series is low (3\%), comparable with some other series. ${ }^{33}$ Indefinite confinement to bed or wheelchair or prolonged mechanical ventilator dependence was not found in our patients. These results indicate that Guillain-Barré syndrome is a disease with a good prognosis in patients who survive the acute stage. On the other hand, FS is not always benign although it has been considered to be a disease with a uniformly benign course. ${ }^{4}$ Persistent disability due to ataxia did occur in our two patients with FS.

Previous studies on Guillain-Barré syndrome have identified several clinical features in patients associated with a poor outcome. These include old age, rapid progression of the illness, and ventilator dependence. ${ }^{14}{ }^{34-36}$ Electrophysiological studies have been reported to be more precise in predicting a poor prognosis; the criteria used previously included mean CMAP amplitude $\leqslant 20 \%{ }^{34}$ or $10 \%{ }^{36}$ of lower limit of normal, and compound abductor pollicis brevis muscle action potentials being absent or $<1 \mathrm{mV} .^{14}{ }^{35}$ Appreciable short term benefits from plasmapheresis as shown by the decrease in the duration of artificial ventilation, the time to onset of motor recovery, and the time to walk independently, have been noted..$^{33} 343738$ In addition, a long term beneficial effect from plasmapheresis was seen in one study. ${ }^{39}$ In our study, three features correlated with a poor outcome: ventilator dependence, a low mean CMAP amplitude ( $\leqslant 10 \%$ of lower limit of normal), and age greater than 40 years. Considerable clinical improvement shortly after the beginning of plasmapheresis was found in most of our patients who received this treatment. However, a long term beneficial effect of plasmapheresis was not detected.

1 Asbury AK, Arnason BG, Adams RD. The inflammatory lesion in idiopathic polyneuritis. Its role in pathogenesis. Medicine 1969;48:173-215.

2 Brown WF, Feasby TE. Conduction block and denervation in Guillain-Barré polyneuropathy. Brain 1984;107:219-39.

3 Albers JW, Donofrio PD, McGonagle TK. Sequential electrodiagnostic abnormalities in acute inflammatory demyelinating polyradiculoneuropathy. Muscle Nerve 1985;8:528 
4 Fisher M. An unusual variant of acute idiopathic polyneuritis (syndrome of ophthalmoplegia, ataxia, and areflexia). $N$ tis (syndrome of ophthalmop.

5 Feasby TE, Gilbert JJ, Brown WF, Bolton CF, Hahn AF, Koopman WF, Zochodne DW. An acute axonal form of Guillain-Barré polyneuropathy. Brain 1986;109:1115-26.

6 McKhann GM, Cornblath DR, Griffin JW, Ho TW, Li CY, Jiang Z, et al. Acute motor axonal neuropathy: a frequent cause of acute flaccid paralysis in China. Ann Neurol 1993; 33:333-42

7 Ho TW, Mishu B, Li CY, Gao CY, Cornblath DR, Griffin JW, et al. Guillain-Barré syndrome in northern China. Relationship to Campylobacter jejuni infection and antiglycolipid antibodies. Brain 1995;118:597-605.

8 Griffin JW, Li CY, Ho TW, Tian M, Gao CY, Xue P, et al. Pathology of the motor-sensory axonal Guillain-Barré syndrome. Ann Neurol 1996;39:17-28.

9 Arnason BGW, Soliven B. Acute inflammatory demyelinating polyradiculoneuropathy. In: Dyck PJ, Thomas PK, ing polyradiculoneuropathy. In: Dyck PJ, Thomas PK, Griffin JW, Low PA, Poduslo JF, eds. Peripheral neuropathy.

10 Hughes RAC, Newsom-Davis JM, Perkin GD, Pierce JM. Controlled trial of prednisolone in acute polyneuropathy. Lancet 1978;2:750-3.

11 Ropper AH, Wijdicks EFM, Truax BT. Guillain-Barré syndrome. Philadelphia: FA Davis, 1991

12 Cornblath DR, Mellits ED, Griffin JW, McKhann GM, Albers JW, Miller RG, et al, The Guillain-Barré Syndrome Study Group. Motor conduction studies in Guillain-Barre syndrome: description and prognostic value. Ann Neurol 1988;23:354-9.

13 Asbury AK, Cornblath DR. Assessment of current diagnostic criteria for Guillain-Barre syndrome. Ann Neurol 1990; 27 (suppl):S21-4.

14 Sedano MJ, Calleja J, Canga E, Berciano J. Guillain-Barré syndrome in Cantabria, Spain. An epidemiological and clinical study. Acta Neurol Scand 1994;89:287-92.

15 Rees JH, Soudain SE, Gregson NA, Hughes RAC. Campylobacter jejuni infection and Guillain-Barré syndrome. $N$ lobacter jejuni infection and

16 Masucci EF, Kurtzke JF. Diagnostic criteria for the Guillain-Barré syndrome. An analysis of 50 cases. 7 Neurol Guillain-Barré syndro

17 Larsen JP, Kvåle G, Nyland H. Epidemiology of the Guillain-Barré syndrome in the county of Hordaland, Western Norway. Acta Neurol Scand 1985;71:43-7.

18 Halls J, Bredkjær C, Friis ML. Guillain-Barré syndrome: diagnostic criteria, epidemiology, clinical course, and prognosis. Acta Neurol Scand 1988;78:118-22.

19 Boucquey D, Sindic CJM, Lamy M, Delmée M, Tomasi JP, Laterre EC. Clinical and serological studies in a series of 45 patients with Guillain-Barré syndrome, $\mathcal{f}$ Neurol $\mathrm{Sci}$ 1991;104:56-63.

20 Jiang GX, de Pedro-Cuesta J, Fredrikson S. Guillain-Barré syndrome in South-West Stockholm, 1973-91, 1. Quality of registered hospital diagnoses and incidence. Acta Neurol Scand 1995;91:109-17.

21 Soffer D, Feldman S, Alter M. Epidemiology of GuillainBarré syndrome. Neurology 1978;28:686-90.
22 Hankey GJ. Guillain-Barré syndrome in West Australia, 1980-1985. Med F Aust 1987;146:130-3.

23 Wint 1985 . Med f A Ast 1987;146:130-3. West Australia, Barré syndrome in Oxfordshire. O f Med 1990;77:1297304.

24 Färkkilä M, Kinnunen E, Weckström P. Survey of GuillainBarré syndrome in southern Finland. Neuroepidemiology 1991;10:236-4

25 Hart DE, Rojas LA, Rosário JA, Recalde H, Román GC. Childhood Guillain-Barré syndrome in Paraguay, 1990 to 1991. Ann Neurol 1994;36:859-63.

26 Al-Din AN, Anderson M, Bickerstaff ER, Harvey I. Brainstem encephalitis and the syndrome of Miller Fisher. A clinical study. Brain 1982;105:481-95.

27 Barontini F, Sitá D. The nosological position of Fisher's syndrome (ophthalmoplegia, ataxia and areflexia). $\mathcal{F}$ Neurol 1983;229:33-44.

28 Petty RKH, Duncan R, Jamal GA, Hadley D, Kennedy PGE. Brainstem encephalitis and the Miller Fisher syndrome. 7 Neurol Neurosurg Psychiatry 1993;56:201-3.

29 Al-Din ASN, Anderson M, Eeg-Olofsson, Trontelj JV. Neuro-ophthalmic manifestations of the syndrome of ophNeuro-ophthalmic manifestations of the syndrome of ophpatients. Acta Neurol Scand 1994;89:87-94.

30 Shuaib A, Becker WJ. Variants of Guillain-Barré syndrome: Miller Fisher syndrome, facial diplegia and multiple cranial nerve palsies. Can f Neurol Sci 1987;14:611-6.

1 Guiloff RJ. Peripheral nerve conduction in Miller Fisher syndrome. $\mathcal{F}$ Neurol Neurosurg Psychiatry 1977;40:801-7.

32 Jamal GA, Ballantyne JP. The localization of the lesion in patients with acute ophthalmoplegia, ataxia and areflexia
(Miller Fisher syndrome). A serial multimodal neurophysiological study. Brain 1988;111:95-114.

33 Guillain-Barré Syndrome Study Group. Plasmapheresis and acute Guillain-Barré syndrome. Neurology 1985;35:1096104.

34 McKhann GM, Griffin JW, Cornblath DR, Mellits ED, Fisher RS, Quaskey SA, The Guillain-Barré Syndrome Study Group. Plasmapheresis and Guillain-Barré syndrome: analysis of prognostic factors and the effect of syndrome: analysis of prognostic factors and th

35 Winer JB, Hughes RAC, Osmond C. A prospective study of acute idiopathic neuropathy. I. Clinical features and their prognostic value. I Neurol Neurosurg Psychiatry 1988;51: 605-12.

36 Miller RG, Peterson GW, Daube JR, Albers JW. Prognostic value of electrodiagnosis in Guillain-Barré syndrome. Muscle Nerve 1988;11:769-74.

37 French Cooperative Group on Plasma Exchange in Guillain-Barré Syndrome. Efficiency of plasma exchange in Guillain-Barré syndrome: role of replacement fluids. Ann Neurol 1987;22:753-61.

38 Smith GDP, Hughes RAC. Plasma exchange treatment and prognosis of Guillain-Barré syndrome. $Q \mathcal{F}$ Med 1992;85: 751-60.

39 French Cooperative Group on Plasma Exchange in Guillain-Barré Syndrome. Plasma exchange in GuillainBarré syndrome: one-year follow-up. Ann Neurol 1992;32: 94-7. 